\title{
Aplicación de minijuegos en Física y Química de Bachillerato
}

\author{
Felipe QuinTANAL PÉREZ \\ Colegio marista "La Inmaculada" de Granada \\ fqyfqyfq@gmail.com
}

\begin{abstract}
Resumen:
La investigación descrita se efectuó durante el curso 2012-2013 con alumnos de $1^{\circ}$ de Bachillerato en las modalidades sanitaria y tecnológica. Se fundamenta en el empleo de minijuegos educativos relacionados con la materia de Física y Química. Los alumnos se organizaron por equipos de trabajo, eligiendo y analizando los minijuegos, elaborando una revista digital, una fotografía interactiva, un test multimedia y un cartel de presentación. Estas actividades fueron recogidas en la red Edmodo, así como la autoevaluación de los alumnos, con gran satisfacción por su parte.
\end{abstract}

Palabras clave: Minijuegos, Bachillerato, Física y Química, Edmodo, Herramientas Web 2.0.

\section{Application of mini-games in Physics and Chemistry of High School.}

\begin{abstract}
:
The described research was carried out during the academic year 2012-2013 with students of 1st Bachelor in health and technological modes. It is based on the use of educational mini-games, related to the subject matter of Physics and Chemistry. The students were organized by teams, choosing and analyzing the mini-games, developing a digital magazine, a photo interactive, a multimedia test and a poster presentation. These tasks were collected in Edmodo network, as well as the self-assessment of the students, with great satisfaction on their part.
\end{abstract}

Key words: Mini-games, High School, Physics and Chemistry, Edmodo, Web 2.0 Tools.

\section{Referencia normalizada:}

Quintanal Pérez, F. (2013) Aplicación de minijuegos en Física y Química de Bachillerato. Historia y Comunicación Social. Vol. 18 Nº Especial Octubre. Págs. 411-420.

Sumario: 1. Introducción. 1.1. Origen de la investigación. 1.2. Fundamentación y contexto de la investigación. 1.3. Videojuegos: definición y características. 1.4. Beneficios pedagógicos de los videojuegos. 2. Metodología empleada. 3. Desarrollo efectuado. 3.1. Etapa inicial. 3.2. Etapa intermedia. 3.3. Etapa final. 4. Resultados obtenidos. 4.1. Productos realizados por los alumnos. 4.2. Valoraciones efectuadas por los estudiantes. 4.3. Valoración del profesor. 5. Conclusiones. 6. Referencias bibliográficas. 


\section{Introducción}

\subsection{Origen de la investigación}

El origen de esta investigación se ancla en dos raíces poderosas. La primera recoge las lecturas previas efectuadas que han suscitado una serie de nuevas preguntas y nuestro interés por generar nuevas ideas. La segunda ha sido nuestra propia experiencia, que desde el conocimiento del contexto en el que nos movemos y de los hechos educativos que acaecen, nos ha permitido sugerir respuestas a los problemas detectados en la praxis.

Secundariamente, la investigación presenta también un fundamento metodológico, pues desde hace años, las asignaturas relacionadas con la ciencia escolar son cuestionadas por la didáctica empleada por el profesorado, el interés que suscita en los alumnos y por el rendimiento académico obtenido.

En último lugar, aparece una razón emotiva como es nuestro cariño personal hacia la Ciencia, lo que nos dirige hacia el compromiso en la búsqueda de medios y de acciones educativas que puedan aportar soluciones en los nuevos escenarios educativos.

\subsection{Fundamentación y contexto de la investigación}

El fundamento de nuestra investigación se apoya en un hecho incuestionable al cual se enfrenta la didáctica de la Física y Química y la enseñanza-aprendizaje de esta ciencia en la escuela. Este hecho consiste en la dificultad que presentan los alumnos para acercarse a una visión holística de la materia citada y descubrir su funcionamiento e implicación en muchos procesos cotidianos de sus vidas.

Además, el propio sistema educativo no ayuda a la mejora de esta tarea. El uso de pedagogías tradicionalmente expositivas, la escasa relación entre los hechos científicos y la vida cotidiana del alumno, los currícula excesivamente saturados, el uso insuficiente de las TIC, la huida en muchos casos del trabajo en el laboratorio, el limitado número de horas lectivas, la excesiva orientación en clase para aprobar exámenes, la tipología del profesorado constituyen algunos factores influyentes que ratifican al estudiante en su percepción de la Ciencia como algo fragmentado, desconectado y con poca implicación en su vida ordinaria.

Con objeto de minimizar el influjo de estos agentes se ha situado el problema descrito en nuestra investigación dentro del contexto de un centro privado-concertado en el que desempeña su labor el investigador y la etapa escogida para ser consolidada, Bachillerato. El curso escogido ha sido $1^{\circ}$ y los alumnos, los pertenecientes a las modalidades de salud y científico-tecnológica. 


\subsection{Videojuegos: definición y características}

Cuando se desea definir un videojuego, éste se puede estimar desde múltiples perspectivas. Una de ellas consiste en escrutarlo como un programa informático interactivo destinado al entretenimiento, que puede funcionar desde diversos dispositivos como pueden ser ordenadores, consolas, teléfonos móviles, tablets, etc.; que integra audio y vídeo y permite disfrutar de experiencias que, en muchos casos, serían muy difíciles de reproducir en la realidad. En consecuencia, los videojuegos representan una alternativa a los relatos cinematográficos o televisivos tradicionales.

Por otro lado, un minijuego en Flash se puede considerar como un tipo de videojuego que se caracteriza por grados de complejidad y sofistificación sucintos, lo que deviene en una enorme sencillez.

Dada la escasez de bibliografía sobre los minijuegos y la consideración citada se estudiarán dentro del grupo de los videojuegos; por tanto, en este subapartado y en el siguiente nos referiremos en general a éstos al ser más generales y haber sido abordados intensivamente desde principios del siglo XXI.

El videojuego se caracteriza porque "su estructura narrativa es muy variada siendo frecuentes aquellos cuyos argumentos se basan en la apología, la parábola, la alegoría, la crónica, los relatos de viaje, los cuentos clásicos, los mitos, los relatos oníricos, los ritos iniciáticos y los denominados juegos de rol" (Ortega Carrillo, 2001: 1).

Si se desea encontrar el núcleo de los videojuegos habrá que buscar en el tipo de narración audiovisual y en su naturaleza hipermedia. La historia narrada linealmente en la que existía una introducción, un argumento y un final o desenlace se ha refinado dando paso a la historia caleidoscópica en la que el jugador moviliza distintas opciones, cada una de las cuales abre una puerta a la intriga y al misterio.

Multitud de estudios realizados muestran la adhesión de niños y jóvenes al uso de videojuegos en su tiempo libre. Esta capacidad enorme de atracción que presentan los videojuegos se puede justificar en base a diversos factores. Se pueden destacar: la índole psico-emocional de este tipo de historias, la simbología subliminal que suele contener, el alto grado de interactividad al que accede el jugador y el enorme poder seductor de las imágenes y de los sonidos tridimensionales.

Desde este espacio lúdico original del videojuego se ha saltado a la apertura de las posibilidades de aplicación en el ámbito educativo. No obstante, hay una escasa producción oficial de productos recreativos digitales, a pesar del énfasis concedido a las posibilidades de enseñar por parte de las instituciones públicas.

Este hecho pone en evidencia la limitación de la definición inicial de videojuego y, además, la comprensión equivocada de las dimensiones de enseñanza y diversión, unidas en un solo producto.

El panorama internacional, sin embargo, apuesta por el aprovechamiento didáctico de los videojuegos, su reconocimiento como producto cultural y la ampliación de las características del mercado: 


\begin{abstract}
"No continuemos buscando todos el mismo mercado o realizando juegos para el mismo tipo de jugador, hay todavía una cantidad gigante de personas en el mundo que no juegan videojuegos y que no consiguen una oferta de videojuegos que se ajuste a sus intereses particulares. Pienso que el mejor ejemplo de esta filosofía fue lo que ocurrió con el Wii: mientras Sony y Microsoft estaban enfocados en la carrera por lograr captar el mercado de "hardcore gamers", Nintendo se metió por una dirección totalmente diferente y le apostó a la interactividad y a incluir en el mercado jugadores que antes jamás se hubiesen sentado (o pararse en el caso del Wii) enfrente de una consola de videojuegos, el resultado de esto ya todos lo conocemos y, nos guste o no el Wii y sus juegos, hay una verdad absoluta que nadie puede discutir: el mercado es ahora más grande" (Enrique Fuentes, 2009).
\end{abstract}

\title{
1.4 Beneficios pedagógicos de los vídeojuegos
}

Los videojuegos incorporan diversos beneficios didácticos. Por ejemplo, se pueden desarrollar destrezas cognitivas, espaciales, motoras y mejorar las destrezas en TIC. También se pueden enseñar hechos (conocimientos, memorización, repeticiones), principios (relación causa-efecto) y resolver problemas complejos; incrementando así la creatividad o aportando ejemplos prácticos de conceptos y reglas que son difíciles de ilustrar en el mundo real. Pueden ser ventajosos a la hora de realizar experimentos peligrosos en la vida real, como por ejemplo, el uso de compuestos químicos tóxicos.

Aunque no todos los videojuegos se diseñan con características pedagógicas, sí incluyen cualidades didácticas para estimular y promover las destrezas cognitivas de los alumnos.

Las premisas de los videojuegos consisten en aprender, memorizar, colaborar, explorar o conseguir información adicional para avanzar. Jugando se aprende y una de las ventajas predominantes de los estudiantes radica en la capacidad que poseen para aprender en ámbitos sugerentes.

Los videojuegos fomentan una colaboración real entre jugadores $\mathrm{y}$, en cierta manera, se asemejan a los entornos de aprendizaje o de trabajo colaborativo, pues se comparte información y se aprende de y con los demás.

Como último beneficio del uso de videojuegos se debe indicar el impacto emocional de éstos en los jugadores, la mejora de su autoestima si son capaces de ganar y la participación en actividades sociales.

\section{Metodología empleada}

La propuesta educativa se ha realizado durante el curso 2012-2013 y se ha dirigido a 43 alumnos de $1^{\circ}$ de Bachillerato de las modalidades sanitaria (24 alumnos) y científico-tecnológica (19 alumnos) que seguían la asignatura de Física y Química. Por sexo, la distribución en la modalidad sanitaria fue de 9 alumnas y de 15 alumnos, 
mientras que en la modalidad científico-tecnológica fue de 2 alumnas y 17 alumnos. En total, 11 alumnas y 32 alumnos. De los 43 estudiantes, 42 provenían de cuarto de E. S. O., de los cuales dos llegaban de centros diferentes y uno repetía primero de bachillerato.

El diálogo con los estudiantes condujo el proyecto hacia el trabajo con los videojuegos y más concretamente, con los minijuegos. Originariamente se supuso que estarían más disponibles y serían más fáciles de relacionar con la temática del curso de Física y Química. Rápidamente se pusieron de acuerdo para usar herramientas Web 2.0 gratuitas para la ejecución de las diversas actividades.

Para que el proyecto no fuese muy complejo, se dividió en tres etapas a lo largo del curso, distribuyéndose las diversas tareas entre las mencionadas etapas. Los alumnos trabajaron por parejas, excepto dos equipos que estaban constituidos por tres componentes.

La comunicación y gestión de las tareas se realizó a través de la red social de microbbloging Edmodo, red privada y gratuita. En palabras del autor Edmodo "permitió establecer asignaciones, el envío de las tareas, su calificación, la realización de encuestas, la compartición de todos los trabajos y la comunicación profesor-alumno y entre alumnos" (Quintanal, 2012: 224).

\section{Desarrollo efectuado}

El proyecto se dividió en tres etapas o fases. La secuenciación de actividades y su cronología se explican a continuación.

\subsection{Etapa inicial}

Correspondió con el primer trimestre del curso 2012-2013. Se les propuso que escogieran un minijuego de Física y otro de Química de una lista propuesta por el profesor, así como que buscaran otro minijuego adicional. Para evitar desorientaciones se les indicó que descartaran minijuegos de contenidos violento, sexista, discriminatorio, que incitasen al juego de azar o apuestas, que contuviesen lenguaje soez y que estimulasen el miedo o el consumo de drogas.

Los tres minijuegos seleccionados fueron sometidos al examen de cada equipo según un modelo que se les suministró en Word y que, una vez relleno, debieron convertir a pdf. En esta etapa clasificaron y analizaron las consideraciones técnicas y contextuales de los minijuegos.

Además, debieron elaborar una pequeña guía en español sobre cómo se juega y algunos "trucos" para avanzar más en cada minijuego. Todo ello lo presentaron utilizando el formato de revista digital mediante el software Issuu. 


\subsection{Etapa intermedia}

Concernió al segundo trimestre del curso 2012-2013. Se continuó con el análisis emprendido en la etapa anterior, completándolo con las consideraciones pedagógicas, la evaluación del minijuego y la recomendación final según una plantilla propuesta. El documento generado también se convirtió a pdf.

Además, los equipos seleccionaron y representaron uno de los minijuegos analizados mediante una fotografía interactiva. El software utilizado fue Thinglink.

\subsection{Etapa final}

Se relacionó con el tercer trimestre del curso 2012-2013. Los alumnos realizaron un test multimedia, de opción múltiple, basado en uno de los minijuegos seleccionados, empleando la herramienta MyStudiyo; un cartel de presentación del trabajo realizado a lo largo del curso mediante piZap y el cuestionario de autoevaluación, que se suministró en Word y, una vez relleno, se convirtió a pdf.

El cuestionario constaba de 14 ítems, de los que 13 eran puntuables de 1 a 5 y el último de redacción libre. De los 13 ítems, seis eran relativos a las tareas efectuadas, cinco a las herramientas tecnológicas utilizadas, 1 a la temática seguida y otro sobre la satisfacción personal de las actividades desarrolladas.

Todas las tareas propuestas fueron alojadas en Edmodo como respuesta a las asignaciones efectuadas por el profesor.

\section{Resultados obtenidos}

Los trabajos realizados presentan una doble naturaleza; de un lado, los relacionados con la construcción del conocimiento, desarrollados por los alumnos y, del otro, las valoraciones de las tareas efectuadas por los estudiantes.

\subsection{Productos realizados por los alumnos}

Cada equipo de trabajo, como se infiere del epígrafe 3, ha seleccionado y analizado tres minijuegos educativos, relacionados con la Física y/o la Química, lo que ha supuesto un total de 51 minijuegos analizados; ha elaborado una revista digital; una imagen representativa de uno de los minijuegos seleccionados, conteniendo, al menos, cuatro interactividades; un test multimedia basado en alguno de los minijuegos analizados, con un tope mínimo de ocho cuestiones de opción múltiple; un cartel de presentación del proyecto realizado y han rellenado individualmente los cuestionarios de autoevaluación.

Todas estas tareas han sido alojadas en Edmodo y han podido ser visualizadas y comentadas por los propios alumnos. 
Se puede apreciar un detalle del análisis de un minijuego (Figura 1).

Figura 1: Fragmento del guión para el análisis efectuado sobre un minijuego.

Actrividades Fisica - Quimica: MYYF .

\section{GUÍA DE ANÁLISIS Y EVALUACIÓN DE MINIJUEGOS - 2}

ALUMNOS/AS: Armando Alejandro $=$ Alesús

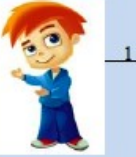

Por favor, rellena el cuestionario y envialo a la plataforma en formato pdf

\begin{tabular}{|c|c|c|c|c|c|}
\hline \multicolumn{6}{|c|}{ ANÁLISIS Y EVALUACIÓN DE MINIJUEGOS (Continuación) } \\
\hline \multicolumn{6}{|l|}{ NOMBRE DEL MINIJUEGO: Physics cup 2} \\
\hline \multicolumn{6}{|l|}{ DIRECCIÓN WEB: http://www.pepijuegos.com/juegos/physics-cup-2 } \\
\hline \multicolumn{6}{|c|}{ CLAVE: 1: Baja; 2: Regular; 3: Alta; 4: Muy alta; 5: Excelente. Marca con una X la puntuación que asignas a cada } \\
\hline La relación del minijuego con el contenido educativo de Física y lo Química es & & & $\mathrm{X}$ & & \\
\hline La definición de los objetivos del minijuego es & & & & $\mathrm{X}$ & \\
\hline $\begin{array}{l}\text { La claridad de la progresión del jugador en el minijuego mediante marcadores o barras } \\
\text { de progresión es }\end{array}$ & & $\mathrm{X}$ & & & \\
\hline La calidad de los comentarios, consejos prácticos y ayuda al jugador es & & $\mathrm{X}$ & & & \\
\hline \multicolumn{6}{|l|}{ 5. Evaluación del minijuego } \\
\hline \multicolumn{6}{|l|}{ Contesta brevemente a cada item } \\
\hline 5.1. Comprensión general del minijuego & & & & & \\
\hline
\end{tabular}

Fuente: Elaboración propia

A continuación se muestra un ejemplo de cartel realizado como presentación del proyecto sobre minijuegos (Figura 2).

Figura 2: Ejemplo de cartel de presentación del proyecto efectuado sobre minijuegos.

Fuente: Elaboración propia

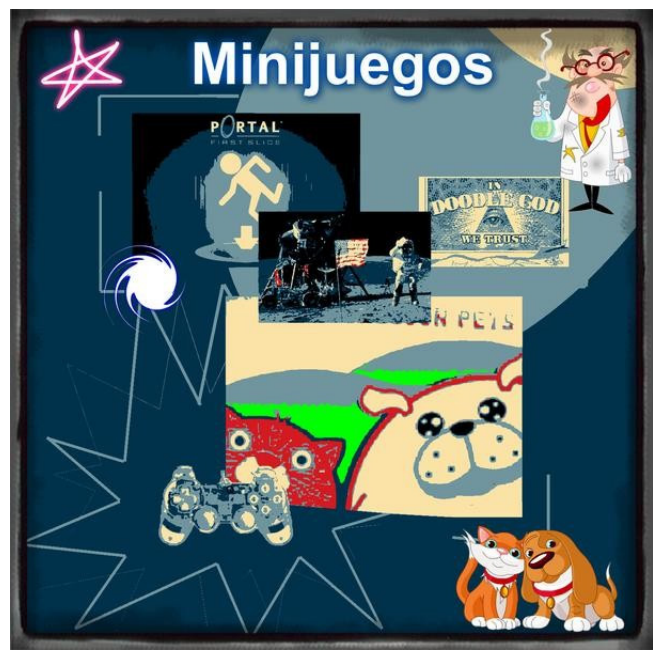




\subsection{Valoraciones efectuadas por los estudiantes}

Se obtuvieron tras la realización del cuestionario elaborado al efecto.

El bloque de ítems relacionado con la experiencia de efectuar las diversas tareas fue valorado muy positivamente, aunque se produjeron leves diferencias.

Se pueden apreciar las medias globales obtenidas en las diferentes tareas (Figura $3)$.

Figura 3: Medias obtenidas para las diversas tareas.

\begin{tabular}{|c|c|c|c|}
\hline TAREAS / MEDIAS & ALUMNOS & ALUMNAS & GLOBAL \\
\hline SELECCION DE MINIJUEGOS & 3,95 & 3,57 & 3,86 \\
\hline ANÁLISIS - 1 & 3,65 & 4,00 & 3,75 \\
\hline ANÁLISIS - 2 & 3,40 & 3,57 & 3,43 \\
\hline FOTOGRAFÍ INTERACTIVA & 4,45 & 3,71 & 4,29 \\
\hline TEST MULTIMEDIA & 4,05 & 4,29 & 4,11 \\
\hline CARTEL DE PRESENTACIÓN & 3,85 & 3,29 & 3,75 \\
\hline
\end{tabular}

Fuente: Elaboración propia

Las tareas más valoradas, considerando la muestra total, fueron la realización de la fotografía interactiva y del test multimedia. La que menos, el análisis de los minijuegos en sus consideraciones pedagógicas y de evaluación, que constituyó la segunda parte del análisis que realizaron los grupos de trabajo.

Por sexo, las alumnas difirieron de la tendencia general al valorar más el test multimedia y la realización del análisis en sus consideraciones técnicas, contextuales y clasificatorias, lo que constituyó la primera parte del análisis, siendo la tarea menos apreciada la realización del cartel de presentación.

A continuación se expone las medias obtenidas sobre la valoración aportada por los estudiantes para las herramientas utilizadas (Figura 4).

Figura 4: Medias obtenidas para las diversas herramientas.

\begin{tabular}{|c|c|c|c|}
\hline HERRAMIENTAS / MEDIAS & ALUMNOS & ALUMNAS & GLOBAL \\
\hline ISSUU & 4,50 & 3,71 & 4,32 \\
\hline THINGLINK & 4,25 & 4,57 & 4,36 \\
\hline MYSTUDIYO & 4,05 & 4,00 & 4,03 \\
\hline PIZAP & 3,75 & 3,71 & 3,71 \\
\hline EDMODO & 4,50 & 3,71 & 4,32 \\
\hline
\end{tabular}

Fuente: Elaboración propia

En conjunto, las herramientas más valoradas fueron Thinglink, aplicación para realizar fotografías interactivas; Edmodo, como almacén de tareas y canal de comuni- 
cación e Issuu, para la realización de la revista digital. La menos valorada fue piZap, herramienta para tratar imágenes.

Por sexo, las alumnas nuevamente no concordaron con la tendencia general al ponderar MyStudiyo en mayor valor que los alumnos, coincidiendo en su apreciación por Thinglink, Edmodo, piZap e Issuu, aunque en menor cuantía que los estudiantes masculinos. La excepción se asentó en Thinglink, donde la valoración femenina fue superior a la masculina. A diferencia de los alumnos, no aparece una tendencia clara sobre la preferencia de alguna herramienta.

Con respecto a la valoración de la temática tratada, los minijuegos educativos, la media manifestada por los discentes fue de 3,93; siendo más alta para los alumnos, 4,00 que para las alumnas, 3,57.

En último lugar se reseña que la satisfacción personal de los estudiantes por las tareas efectuadas fue muy alta. Así, la media general obtenida fue de 4,32. Por sexo, los alumnos consiguieron una media de 4,40 y las alumnas de 4,00.

\subsection{Valoración del profesor}

En general, muy positiva. Según los comentarios expuestos por los estudiantes y de los eventos observados por el profesor se aprecia coincidencia en la valoración global de la experiencia.

Como aspectos importantes se resaltan:

- El incremento de la motivación del alumnado, inferido de la mejora del rendimiento académico y del aumento de la autoconfianza de los estudiantes.

- La mejora de distintas competencias como las sociales, las intelectuales (expresadas mediante la habilidad para cooperar y explorar, la independencia, la responsabilidad, la iniciativa y el dinamismo), las espacio-temporales (articuladas a través de los reflejos) y la concentración, así como la competencia digital.

\section{Conclusiones}

Se pueden citar las siguientes:

- La utilización de minijuegos favorece el aumento de la motivación de los estudiantes, así como el afianzamiento de su autonomía, autoconfianza y autoestima.

- El proyecto ejecutado contribuye a cumplir con los objetivos educativos en términos de contenidos, competencias (sobre todo, sociales) y valores.

- Favorece la consideración de los ritmos de aprendizaje, del trabajo en equipo y del progreso de las competencias intelectuales. 
- Desarrolla y potencia las destrezas TIC del alumnado y la consideración de éstos como creadores de contenido científico que pueden compartir con sus compañeros.

- El empleo de minijuegos es aplicable a otros niveles y áreas. Los niveles pueden ser $3^{\circ}$ y $4^{\circ}$ de E. S. O, Educación Primaria y Educación Infantil. Las áreas implicadas en Educación Primaria y Secundaria pueden ser Lengua (oficial y extranjera), Historia, Geografía, Matemáticas y Biología.

\section{Referencias bibliográficas}

FELICIA, P. (2009): Videojuegos en el aula: manual para docentes. European Schoolnet. Bruselas. Versión electrónica. Disponible en:

http://games.eun.org/upload/GIS_HANDBOOK_ES.pdf. [1-10-2013].

FUENTES, E. (2009): "Casual connect y la oportunidad de los videojuegos casuales". En Videojuegos en Venezuela. Disponible en:

http://videojuegosve.blogspot.com.es/2009/10/casual-connect-y-la-oportunidad-delos.html. [1-10-2013].

ORTEGA CARRILLO, J. A. (2001): "Análisis crítico de los valores que transmiten los videojuegos: Descubriendo su potencial seductor de naturaleza subliminal".

En Seminario Virtual UNESCO sobre Educación y Tecnologías de la Información. Universidad de Granada. Disponible en:

http://www.ugr.es/ sevimeco/documentos/iaplicadas/a_valores.doc. [31-9-2013].

QUINTANAL PÉREZ, F. (2012): "Integración de podcast en la enseñanza de Física y Química de Bachillerato". En ZAMARRA LÓPEZ, M. M. (coord.): Aplicaciones docentes en el ámbito del EEES. Madrid: Vision Libros. p. 217-233.

SÁNCHEZ, P. (2009, 23 de abril): "El videojuego: producto cultural". En MeriStation. Disponible en:

http://www.meristation.com/pc/reportaje/el-videojuego-producto-cultural/1602655. [1-10-2013].

\section{El autor}

Felipe Quintanal Pérez es Doctor en Ciencias de la Educación por la UNED, Licenciado en Ciencias Químicas por la Universidad Hispalense de Sevilla y Master en Informática Educativa por la UNED. Su labor profesional se desarrolla como profesor de Ciencias en Secundaria y Bachillerato. Como investigador trabaja actualmente en la mejora de los estilos de aprendizaje aplicados a la Física y Química de Bachillerato y de la ESO empleando diversas herramientas Web 2.0 y en Programas de Innovación Docente de la Universidad de Granada, colaborando con el Departamento de Química Analítica de la Facultad de Ciencias. 